\title{
Alpha-Fetoprotein (AFP) and Gastric Cancer: Why is Lethality More Prevalent in AFP-Secreting than Non- Secreting Tumors?
}

\author{
GJ Mizejewski* \\ Division of Translational Medicine, Molecular Diagnostics Laboratory, USA
}

Submission: December 21, 2017; Published: January 08, 2018

"Correspondence Address: GJ Mizejewski, Division of Translational Medicine, Molecular Diagnostics Laboratory, Wadsworth Center, New York State Department of Health, PO Box 509, Empire State Plaza, Albany, NY 12201-0509, USA,

Email: gerald.mizejewski@health.ny.gov

\begin{abstract}
Gastric (stomach) cancer (GC) is the fourth leading cause of cancer deaths worldwide. Alpha-fetoprotein (AFP) secreting gastric cancer $(\mathrm{AFP}(+)$ GC represents an aggressive, less common subtype of stomach cancer exhibiting poor prognosis, low patient survival times, high progression rates, and liver metastases. No standard treatment regimen is presently in practice although multimodal therapies have been employed, while some drug resistance has been encountered in chemotherapy. AFP(+)GC is known to be more lethal than AFP-nonsecreting tumors with a patient median survival time of only 14 months. The reason for the increased mortality and morbidity of AFP-secreting GC has not been completely understood although multiple factors have been forwarded. Such factors include later stage diagnosis, unresectable metastases, rapid tumor growth, high mitotic rates, and elevated cellular expression of growth-promoting proteins. However, in recent years, a better understanding of the physiological (biological) activities of AFP as a growth regulatory cell-signaling factor have emerged. Such studies have established that AFP itself, acting through a cell surface receptor, is a potent tumor growth promoter as demonstrated in the present report.

Keywords: Alpha-fetoprotein; Gastric Cancer; Stomach Tumors; Adenocarcinoma; Survival Times; Liver Metastasis; Lethality; Aggressive Growth

Abbreviations: AFP: Alpha-Fetoprotein; BAX: Bcl2 associated -4- Protein; Bcl2: B-cell Lymphoma-2; CdK6: Cyclin Dependent Kinase-6; C-Met: Hepatocyte Growth Factor; ERB-B2: Human Epidermal Growth Factor Receptor (HER2); GAAD153: Growth Arrest/DNA Damage Inducible Transcription Factor; IAP: Inhibitor of Apoptosis; IF-1R: Insulin Growth Factor-Ireceptor; IGF: Insulin Growth Factor; NCAM: Neural Cellular Adhesion Molecule; PI3K: Phosphoinositol-3-Kinase; PTEN: Phosphatase and Tensin Homolog; VEGF: Vascular Growth Factor; XIAP: X-limited Inhibitor of Apoptosis; HcG: human Chorionic Gonadophin; CEA: Carcinoembryonic Antigen
\end{abstract}

\section{Introduction}

Gastric cancer (GC), a heterogenous adenocarcinoma of the stomach, is the fourth most common cancer and the second cause of cancer death worldwide [1]. Alpha-fetoprotein (AFP) secreting GC $(A F P(+) G C$ is quite different from the conventional stomach cancer (AFP(-) GC) being more aggressive, having lower patient survival times, and prone to liver/lymph node metastasis [2]. Another subtype of GCs, termed the Signet Ring cell gastric carcinoma, originates in the stomach glandular cells, produces mucin and ERBB2 (an oncogenic receptor), and is histopositive for AFP and its cell surface receptor [3]. A third subtype of GC is hepatoid cancer (HC) of the stomach which mimics a hepatocellular carcinoma. The HC is also a high AFP(+) secreting stomach cancer characterized by lymphadenopathy, liver metastasis, and venous tumor thrombosis [4-6].
The AFP(+)GC is an aggressive, highly malignant, metastatic, fast progressing tumor with a poor prognosis. No standard therapy is currently available for patients with $\mathrm{AFP}(+) \mathrm{GC}$ although several multi-modal therapies have been reported [2]. These modalities include surgery, chemotherapeutics, drugs, and interventative and molecular targeted therapies. The chemo-drug therapies have encompassed;
i. Sorfenib;
ii. Oxalipatin;
iii. Paclitaxel;
iv. Irinotecan; 
v. Fluorourical;

vi. Calcium folate; and

vii. Capecitabine $[1,2]$.

AFP (+)GCs further exhibit poor differentiation, detection at late clinical stages, large tumor volume, high rate of invasion and metastasis, and elevated AFP serum levels [7]. Median patient survival time is 14.0 months with a 1.0, 3.0, and 5 year survival rates of 58, 31, and 19\%, respectively [4]. AFP(+)GC constitutes a 1.3 to $15 \%$ incidence of all gastric tumors [2]. In some cases of $\mathrm{AFP}(+) \mathrm{GC}$, elevated levels hCG and CEA have been reported $[2,3]$.

\section{Stomach Cancer and AFP}

AFP is a proven biomarker for hepatocellular carcinoma in the serum of adults. Serum AFP can further serve as a biomarker in other cancers such as testicular seminomas, yolk sac tumors of the ovary, and malignant teratomas [8-10]. Moreover, AFP can also indicate the presence of certain anemias and noncancerous liver diseases including alcoholic cirrhosis and viral hepatitis [11]. Lastly, elevated serum levels of AFP can further accompany the growth of malignant lung and bladder tumors and gastrointestinal cancers of the stomach, pancreas, and colon [12]. Interestingly, the stomach emerges as one of the more common organ cancers which display elevated serum AFP levels $[1,2]$.

The first clinical case of AFP associated with stomach cancer was reported by Boureille and his associates in 1970 [13] followed by a monograph publication by Akai et al. [14]. An additional nine cases of AFP(+)GC were reported by Nishio et al. [15] in 1989 and many cases have since followed. A case of metastatic AFP(+)GC was published by Kodama et al. [16] and a hepatoid subtype was first identified by Ishikawa and Adochi et al. [17]; this was followed by a detailed immunocytochemical analysis in 1988 [18]. It is of interest that various drug treatments and their drug resistance were first described in 2006 while using opachititoxel. It was subsequently suggested but not demonstrated that the $\mathrm{ABC}$ transporter, p-glycoprotein, may be a possible cause of the drug resistance detected in the AFP(+)GC (see below).

The prognosis for AFP(+)GC is much worse than reported for AFP(-)GC patients for many reasons, one of which is the elevated AFP serum levels discussed below. However, other contributing factors to the higher mortality and morbidity rates can be listed as follows. These factors would include:

a. Diagnosis at advanced tumor stage;

b. Unresectable metastasis;

c. Increased cell invasion and migration;

d. High mitotic rates;

e. Active cell proliferation;

\section{f. Rapid tumor progression, and}

g. High cytoplasmic expression of apoptosis, growth promoting factors, and cytoplasmic signaling-related proteins [19].

The reported growth enhancing factors included proteins such as c-Met, VEGF, and IF-1R and cell cycle associated factors such as Cyclin B1, Cyclin D1, and CdK6. The apoptosis-regulating factors encompassed apoptosis inhibiting proteins such as XIAP, BcL2, and BAX, while the signaling-related proteins included Caspase-3, 9, PTEN phosphatase, and PI3K inositol kinases [19]. However, highly elevated serum AFP is the signature trait of these tumors.

\section{Consequences of High Serum AFP Levels Upon GC}

The question may be posed of "why are the AFP(+)GCs reported as more lethal than non-secreting AFP stomach cancers?" To answer this query one must first pursue what growth affects does full-length AFP, an established autocrine growth promoter, have on the tumor cell that secreted it? A search of the biomedical literature readily reveals that $85 \%$ of stomach tumors display the canonical AFP cell surface receptor that can bind and interealize the AFP molecule [20]. The AFP receptor binding and uptake initiates a cascade of signal transduction pathways culminating in enhanced cell growth. Published reports demonstrate that AFP secreted into the blood circulation can amass concentrations ranging from $45 \mathrm{ng} / \mathrm{ml}$ to $200 \mathrm{ng} / \mathrm{ml}$ with some tumors expressing microgram levels of 10 $60 \mathrm{ug} / \mathrm{ml}$ [2]. Thus, the uptake of such AFP levels into gastric tumor cells appears to have immense consequences on cell growth and proliferation.

The underlying molecular mechanism attributed to the poor prognosis and low survival times of AFP(+) compared to AFP(-)GC patients is at present poorly understood. The present report represents an attempt to advance knowledge and aid in elucidating the molecular differences observed in the two types of stomach cancers. At the onset, it must be understood that AFP has two distinct functional forms; the first being classical AFP secreted into the blood circulation [7], while the second is a non-secreted AFP form that resides in the cytoplasm and reacts with transcription factors in normal and tumor cells [21]. The cytoplasmic AFP (cyAFP) differs from secreted AFP in that the former lacks both the amino-terminal signal sequence of 19 amino acids and differs in the glycosylation patterns present in the secreted full length AFP. Both functional forms of AFP must be considered prior to probing the causes of lethality of AFP in GC. First and foremost, it must be emphasized that AFP(+)GCs have been reported to display an increased expression of AFP. high affinity receptors on the surface of the tumor cell membranes; while few, if any, are present on present on benign gastric tissues and stomach cells adjacent to the tumor site $[20,22]$.

AFP has been reported to be an autocrine growth factor that binds to its cognant receptor, activates said receptor, and 
initiates a cascade of downstream signaling events to enhance cell growth [23]. Secondly, a recent report documenting a protein profile of $\mathrm{AFP}(+) \mathrm{GCs}$ using microarray analyses demonstrated that multiple proteins out of 286 tested were found to be differentially expressed between AFP(+) and AFP(-) GCs [19]. The multiple expressed proteins were grouped as follows:

i. Apoptosis regulating proteins (XIAP, Bcl2, Caspases, $\mathrm{BAX})$;

ii. Growth factors and kinase enzymes and receptors (c-Met, IGF-1R, HGF), and the PTEN phosphatase;

iii. Metastasis-associated proteins (cadherins, NCAM1, Calpain);

iv. Cell cycle associated proteins (CdK6, Cyclin-D, Cyclin-B);

v. Cell coating and protective proteins (mucins, MUC2, MUC5AC); and

vi. Solute carrier transporter proteins (cation/anion transporters, sodium/phosphate co-transporters, and calcium/sulfate transporters [19].

The cell surface expression of various mucins (MUC2, MUC5AC) in AFP(+)GC listed above were shown to reflect different levels of differentiation of gastric adenocarcinoma cells [3]. Furthermore, MUC2 is a gastrointestinal mucin that is known to associate with MUC6 to promote tumor progression, while MUC5A is a trypsin inhibitor containing a Von Willebrand domain for binding to extracellular proteins and integrins. It is noteworthy that AFP binds to both of these mucins which activate multiple intracellular phosphoinositol signaling pathways leading to growth enhancement [23].

AFP(+)GCs were also found to express increased amounts of several solute carrier transporter (SCT) proteins compared to AFP(-) tumors [24]. This observation is especially noteworthy in that the SCT proteins are crucial for the uptake of chemotherapeutic drugs such as gencitabins and 5-fluorouricil. Moreover, using AFP-derived peptide fragments in in vitro mRNA microarray analysis, it was demonstrated that down regulation of anion and cation SCT proteins occurred at levels of 6 -fold or more than controls [25]. These latter data signified that AFP peptide segments could reduce the levels of cytoplasmic SCT proteins which are responsible for the uptake of chemotherapeutic drugs in $\mathrm{AFP}(+)$ stomach cancer cells. The above-stated biological activities of AFP in GC cells would notably contribute to the aggressive behavior and poor survival characteristics of this subtype of gastric cancer.

The second biological function exhibited by AFP concerns its presence as an intracellular, non-secreted form (CyAFP) that exhibits multiple cell growth functions [21]. In its intracellular form, CyAFP can dimerize with cell-bound proteins such as cytoplasmic caspases, cell cycle-associated proteins, nuclear receptors (i.e. retinoic acid), apoptosis-inhibiting proteins, PTEN and FN14 growth stimulators, and a growth arrest and DNA damage-indescribable (GAAD153) protein. From microarray protein profiling analyses of $\mathrm{AFP}(+) \mathrm{GC}$, it has been reported that such tumors show increased expression of proteins with which CyAFP can bind, dimerize, and/or interact [19]. Such cytoplasmic proteins include PTEN, Caspase 3, CDK6, cyclins B1, Cyclin-D1, Bcl2, IGF-1, NCAM1, and proteins that inhibit apoptosis (XIAP, IAP). All the above cytoplasmic proteins are engaged in promoting the cell growth and proliferation of gastric cancer cells and could be regulated by AFP peptide fragments.

In summary, documented reports in the biomedical literature have demonstrated multiple biological activities of AFP that could contribute to the aggressiveness of AFP(+)GCs. These activities include the six following characteristics:

a. AFP is a growth promoting autocrine hormone-like protein;

b. AFP receptors are highly expressed on the cell surface of AFP(+)GC cells;

c. Protein profiling of $\mathrm{AFP}(+) \mathrm{GC}$ cells have been shown to display high expression of proteins associated with apoptosis, metastasis, receptor kinases, growth factors, and the cell cycle;

d. AFP can bind and activate stomach and intestinal cell surface mucins involved in cell signaling pathways that enhance tumor cell growth;

e. AFP peptides were found to down-regulate the mRNA of solute carrier transporter (SCT) proteins which function in the cellular uptake of chemo-therapeutic drugs;

f. The presence of cytoplasmic AFP has been demonstrated to bind, dimerize, and interact with intracellular growth promoting factors that contribute to cell growth, proliferation, and metastasis of stomach cancer cells.

\section{Conclusion}

$\mathrm{AFP}(+) \mathrm{GC}$ is an aggressive, poorly differentiated tumor of the stomach often accompanied by unresectable metastatic lesions and elevated AFP serum levels. This AFP-secreting stomach adenocarcinoma differs from the conventional type of gastric cancer by having a poorer prognosis and lower survival time of patients which are attributed to liver metastasis and lymphovascular invasion among other traits $[5,26]$. Moreover, $\mathrm{AFP}(+)$ stomach cancer further exhibits increased mitosis and cell migations, high proliferative rates, and rapid tumor progression [11]. The greater mortality and morbidity of the AFP-secreting tumors may be largely due to the presence of AFP as a growth promoter [10,27]. As discussed above, secreted AFP is an autocrine enhancing growth factor with high binding 
affinity to an AFP receptor residing on the cell surface of multiple tumor types, including gastric tumors [20,28]. In fact, I125 radiolabeled AFP-peptides were found to specifically localize in the stomach at 20 times the blood levels in tumor-bearing mice [29].

In addition, reports in the biomedical literature have demonstrated multiple AFP growth enhancing activities resulting from the inhibition of apoptosis, presence of solute carrier transporter proteins of chemo drugs, mucin interaction, and mRNA down-regulation of cell cycle kinases and checkpoint proteins. Cytoplasmic AFP can further regulate cancer cell growth and proliferation, tumor progression, and metastasis. The future clinical goal of treating of AFP(+) secreting tumors may someday entail the elimination or down-regulated expression of the AFP polypeptide $[10,27]$. This feat could be accomplished by recently published procedures employing inhibitory miRNA for AFP; silencing of AFP synthesis (siRNA) or perhaps new gene modulation and editing procedures meant to eliminate AFP production [30,31]. The knowledge of biochemical procedures which could contribute to rapid demise of $\mathrm{AFP}(+)$ in secreting gastric and other tumors should prompt further investigation into these reported methods. The present study might also serve as an impetus for investigators to pursue potential new and novel treatment options for AFP-secreting gastric cancers. Armed with such information, clinicians may be better equipped to consider additional treatment options for GC patients displaying nonthreatening clinical status.

\section{Disclosure}

Financial-None; no U.S. federal grants were used in the preparation of this paper.

\section{Conflict of Interest}

The author declares that there are no known conflicts of interest in the preparation of this manuscript

\section{References}

1. Sun N, Sun Q, Liu Q Zhang T, Zhu Q, et al. (2016) alpha-fetoproteinproducing gastric carcinoma: A case report of a rare subtype and literature review. Oncol Lett 11: 3101-3104.

2. Fang YU, Wang L, Yang N, Gong X, Zhang YU, et al. (2015) Successful multimodal therapy for an alpha-fetoprotein-producing gastric cancer patient with simultaneous liver metastases. Oncol Lett 10(5): 30213025 .

3. Fujimoto A, Ishikawa Y, Ishii T, Yamada A, Igarashi Y, et al. (2017) Differences between gastric signet-ring cell carcinoma and poorly differentiated adenocarcinoma: A comparison of histopathologic features determined by mucin core protein and trefoil factor family peptide immunohistochemistry. Pathol Int 67(8): 398-403.

4. Liu XM, Chen GQ, Li SL, Zai TS (2015) Hepatoid adenocarcinoma of the stomach: A case report and literature review. Exp Ther Med 9(6): 2133-2136.

5. Ren A, Cai F, Shang YN, Ma ES, Huang ZG, et al. (2014) Gastric hepatoid adenocarcinoma: a computed tomography report of six cases. World ] Gastroenterol 20(40): 15001-15006.

6. Lu S, Ma Y, Sun T, Ren R, Zhang X, et al. (2016) Expression of alpha- fetoprotein in gastric cancer AGS cells contributes to invasion and metastasis by influencing anoikis sensitivity. Oncol Rep 35(5): 29842990.

7. Gong W, Shou D, Gong P (2016) Extremely high expression of serum alpha-fetoprotein level of gastric adenocarcinoma: a rare case with an unexpected well-prognosis. Springer plus 5: 2056.

8. Zuo CT, Ju Q (2015) An analysis of clinical characteristics and prognosis for patients with serum alpha-fetoprotein-positive gastric cancer. Minerva Med 106(4):185-191.

9. Mizejewski GJ (2002) Biological role of alpha-fetoprotein in cancer: prospects for anticancer therapy. Expert Rev Anticancer Ther 2(6): 709-735.

10. Mizejewski GJ (2016) Does alpha-fetoprotein contribute to the mortality and morbidity of human hepatocellular carcinoma? A commentary. J Hepatocell Carcinoma 3: 37-40.

11. Lakhi NA, Mizejewski GJ (2017) Alpha-fetoprotein and Fanconi Anemia: Relevance to DNA Repair and Breast Cancer Susceptibility. Fetal Pediatr Pathol 36(1): 49-61.

12. Mizejewski G (2014) Alpha-fetoprotein as a biomarker for Immunology diseases: relevance to ataxia telangiectasia and related disorders. J Immunodeficiency and Disorder 3(1): 1-12.

13. Bourreille J, Metayer P, Sauger F, Matray F, Fondimare A (1970) Existence of alpha-fetoprotein during gastric-origin secondary cancer of the liver. Presse Med 78(28): 1277-1278.

14. Akai S, Kato K (1973) Serum alpha-fetoprotein-positive stomach cancer. Gann Monographs Cancer Res 14: 149-154.

15. Nishio Y, Urakawa T, Nakamoto M, Yamaguchi M, Tanaka H, et al. (1989) Study of nine cases of alpha-fetoprotein (AFP) producing gastric cancer. j jpn Soc Clin Surg 50: 1176-1180.

16. Kodama T, Kameya T, Hirota T, Shimosato Y, Ohkura H, et al. (1981) Production of alpha-fetoprotein, normal serum proteins, and human chorionic gonadotropin in stomach cancer: histologic and immunohistochemical analyses of 35 cases. Cancer 48(7): 1647-1655.

17. Ishikura H, Fukasawa Y, Ogasawara K, Natori T, Tsukada $Y$, et al. (1985) An AFP-producing gastric carcinoma with features of hepatic differentiation. A case report. Cancer 56(4): 840-848.

18. Ganjei P, Nadji M, Albores-Saavedra J, Morales AR (1988) Histologic markers in primary and metastatic tumors of the liver. Cancer 62(9): 1994-1998.

19. He L, Ye F, Qu L, Wang D, Cui M, et al. (2016) Protein profiling of alphafetoprotein producing gastric adenocarcinoma. Oncotarget $7(19)$ : 28448-28459.

20. Tsuboi S, Taketa K, Nouso K, Fujikawa T, Manabe K, et al. (2006) High level of expression of alpha-fetoprotein receptor in gastric cancers. Tumour Biol 27(6): 283-288.

21. Mizejewski GJ (2015) Nonsecreted cytoplasmic alpha-fetoprotein: a newly discovered role in intracellular signaling and regulation. An update and commentary. Tumour Biol 36(12): 9857-9864.

22. Mizejewski GJ (2011) Review of the putative cell-surface receptors for alpha-fetoprotein: identification of a candidate receptor protein family. Tumour Biol 32(2): 241-258.

23. Mizejewski GJ (2013) Review of the adenocarcinoma cell surface receptor for human alpha-fetoprotein; proposed identification of a widespread mucin as the tumor cell receptor. Tumour Biol 34: 13171336.

24. Shimakata T, Kamoshida S, Kawamura J, Ogane N, Kameda Y, et al. (2016) Immunohistochemical expression profiles of solute carrier transporters in alpha-fetoprotein-producing gastric cancer. Histopathology 69(5): 812-821. 
25. Mizejewski GJ (2011) Mechanism of Cancer Growth Suppression of Alpha-Fetoprotein Derived Growth Inhibitory Peptides (GIP): Comparison of GIP-34 versus GIP-8 (AFPep). Updates and Prospects. Cancers (Basel) 3(2): 2709-2733.

26. He R, Yang Q, Dong X, Wang Y, Zhang W, et al. (2017) Clinicopathologic and prognostic characteristics of alpha-fetoprotein-producing gastric cancer. Oncotarget 8(14): 23817-23830.

27. Mizejewski GJ (2011) Therapeutic use of human alpha-fetoprotein in clinical patients: is a cancer risk involved? Int J Cancer 128(1): 239 242.

28. Mizejewski GJ (2015) The alpha-fetoprotein third domain receptor binding fragment: in search of scavenger and associated receptor targets. J Drug Target 23(6): 538-551.
29. Mizejewski GJ, Mirowski M, Garnuszek P, Maurin M, Cohen BD, et al. (2010) Targeted delivery of anti-cancer growth inhibitory peptides derived from human alpha-fetoprotein: review of an International Multi-Center Collaborative Study. J Drug Target 18(8): 575-588.

30. Li C, Wang S, Jiang W, Li H, Liu Z, et al. (2012) Impact of intracellular alpha fetoprotein on retinoic acid receptors-mediated expression of GADD153 in human hepatoma cell lines. Int J Cancer 130(4): 754-764.

31. Zhu M, Guo J, Xia H, Li W, Lu Y, et al. (2015) Alpha-fetoprotein activates AKT/mTOR signaling to promote CXCR4 expression and migration of hepatoma cells. Oncoscience 2(1): 59-70.

\begin{tabular}{l} 
Your next submission with Juniper Publishers \\
will reach you the below assets \\
- Quality Editorial service \\
- Swift Peer Review \\
- Reprints availability \\
- E-prints Service \\
- Manuscript Podcast for convenient understanding \\
- Global attainment for your research \\
- Manuscript accessibility in different formats \\
( Pdf, E-pub, Full Text, Audio) \\
- Unceasing customer service \\
Track the below URL for one-step submission \\
https://juniperpublishers.com/online-submission.php \\
\hline
\end{tabular}

